\title{
Portfolio decision with a quadratic utility and inflation risk
}

\author{
Byung Hwa Lim and Ho-Seok Lee ${ }^{2^{*}}$
}

${ }^{*}$ Correspondence:

hoseoklee@kw.ac.kr

${ }^{2}$ Department of Mathematics, Kwangwoon University, Seoul, Republic of Korea

Full list of author information is

available at the end of the article

\section{Springer}

\begin{abstract}
This paper considers a portfolio selection problem with a quadratic utility of consumption, which is symmetric with respect to a bliss point. At bliss point, the utility function has its maximum value and further consumption lowers the utility. In the presence of inflation risk, we introduce an inflation-linked index bond to manage the inflation risk and derive explicit expressions for the optimal consumption and portfolios by applying duality method. Based on quantitative results, we see that inflation-linked index bond plays an important role in choosing consumption and portfolio rules.
\end{abstract}

Keywords: Martingale duality method; Portfolio selection; Quadratic utility; Inflation risk; Inflation-linked index bond

\section{Introduction}

A quadratic utility function is widely applied in economic and finance theory. The main advantage of a quadratic utility function is its tractability, and this is the main reason why the quadratic function is applied as an objective function for various optimization problems in economics and finance. In particular, modern investment theory and practice are mainly based on the mean-variance portfolio theory developed by Markowitz [7], where the analysis is based on the quadratic function of portfolio return. The analysts in practice apply the mean-variance-efficient portfolios for asset allocation. Recently, Sharpe [9] showed that the mean-variance portfolio allocation has similar results to that of the expected utility asset allocation if we consider the quadratic utility whose risk aversion is measured by the variance of portfolio return.

In this paper we consider a quadratic utility as an agent's preference. Similar to the traditional utility functions of constant relative risk aversion (CRRA) or constant absolute risk aversion (CARA), the law of diminishing marginal utility still holds for the quadratic utility. The marginal utility, however, can be negative when the consumption level is higher than a bliss point. In other words, above a bliss point, if the agent consumes more he/she reduces utility. Thus, the optimal level of consumption and value function should be constant if the wealth level is greater than the critical level at which the optimal consumption rate is the bliss point. With a quadratic utility function, investment pattern is quite different from that when using other utility functions for a sufficiently high wealth level. For example, for an agent with a quadratic utility investment in the risky asset is zero when the wealth level is larger than the bliss level (see, e.g., Koo et al. [5] or Rho et al. [8]).

(c) The Author(s) 2018. This article is distributed under the terms of the Creative Commons Attribution 4.0 International License (http://creativecommons.org/licenses/by/4.0/), which permits unrestricted use, distribution, and reproduction in any medium, provided you give appropriate credit to the original author(s) and the source, provide a link to the Creative Commons license, and indicate if changes were made. 
Due to the negative marginal utility for higher consumption levels, the quadratic utility seems to be unrealistic. It is worth to note, however, that the quadratic utility is sufficient to explain the agent's economic behavior for the realistic level of wealth. Koo et al. [5] studied the quadratic utility in the presence of subsistence consumption constraint, and Rho et al. [8] investigated the effect of borrowing limits on the consumption and investment of the agent with a quadratic utility.

If the time horizon is long enough, it is persuasive to incorporate the inflation risk into consumption and portfolio selection problem. In our continuous-time model, the time horizon is infinite, and thus we expect that the inflation rate would significantly affect the long-term financial planning. To hedge the inflation risk, we introduce the inflationlinked index bond. In fact, many developed countries already have inflation-linked securities which are traded in the financial market. Especially, Treasury Inflation-Protected Securities (TIPS) which were introduced in 1997 have become the most traded bonds in the US bond market.

In this paper, we provide explicit expressions for consumption and portfolio rules by applying the duality approach. Due to the optimal consumption process, we have to separate the wealth into three regions as in Koo et al. [5] and Rho et al. [8]. In the third region, where the wealth level is above the bliss level, the optimal consumption stays constant as its maximum so the value function is also fixed. To make the value function constant, there should be no risky asset investment. This fact is well-described in Koo et al. [5] and Rho et al. [8]. In the presence of inflation risk, however, even though the value function in the third region is still constant, the optimal investment in an inflation-linked index bond is not fixed. To hedge the inflation risk, all the financial wealth is invested in an inflationlinked index bond and investment in other assets becomes zero in the third region. When the wealth level is below the bliss level, the role of inflation-linked index bond can be different according to market parameters. In particular, for sufficiently large growth rate of the price process, the demand for speculative motive is reflected only on the inflationlinked index bond. For a small growth rate of the price process, however, the demand for speculative motive is distributed into both portfolios of inflation-linked index bond and the risky asset.

There are large strands of literature on the portfolio selection problem with inflation risk, and most studies consider the inflation-linked index bond to manage inflation risk. Fischer [3] investigated the demand for index bond and claimed its introduction. Later, Campbell and Viceira [2] and Brennan and Xia [1] studied the dynamic asset allocation in the presence of inflation and interest risks. Gong and $\mathrm{Li}$ [4] incorporated the subsistence consumption constraint into the model with inflation risk. Recently, Kwak and Lim [6] investigated the effect of inflation risk on life-insurance purchase and provided quantitative analysis of the role of an index bond.

This paper is organized as follows. In Sect. 2, we provide the financial market in the presence of inflation risk. We introduce an inflation-linked index bond to hedge the inflation risk. In Sect. 3, we state the optimization problem with a quadratic utility function and seek to derive explicit solutions to the optimization problem by applying the duality approach. In Sect. 4, explicit forms of the optimal consumption and portfolios are given, and we provide some implications of the results in Sect. 5. Finally, Sect. 6 concludes. 


\section{Model}

In our continuous-time model, we assume that the financial market consists of a riskless asset (money market account), a risky asset (stock), and an inflation-linked index bond. Let us denote by $B_{t}$ the price of the riskless asset at time $t$ and assume that the riskless asset earns a constant rate of return $R>0$ as follows:

$$
\frac{d B_{t}}{B_{t}}=R d t
$$

The price of the risky asset at time $t$, denoted by $S_{t}$, evolves according to the following geometric Brownian motion:

$$
\frac{d S_{t}}{S_{t}}=\mu_{S} d t+\sigma_{S} d W_{1, t}
$$

where $\mu_{S}>R>0$ and $\sigma_{S}>0$ are constants; $W_{1, t}$ is a standard Brownian motion on a probability space $(\Omega, \mathcal{F}, \mathbb{P})$. Let us denote by $I_{t}$ the price of the inflation-linked index bond at time $t$, which evolves according to the following equation:

$$
\frac{d I_{t}}{I_{t}}=r d t+\frac{d P_{t}}{P_{t}}
$$

where $r>0$ is the real interest rate and $P_{t}$ is the price level at time $t$. We assume that $P_{t}$ proceeds according to the following geometric Brownian motion:

$$
\frac{d P_{t}}{P_{t}}=\mu_{P} d t+\sigma_{P}\left(\rho d W_{1, t}+\sqrt{1-\rho^{2}} d W_{2, t}\right)
$$

where $W_{2, t}$ is another standard Brownian motion on the probability space $(\Omega, \mathcal{F}, \mathbb{P})$, which is independent of $W_{1, t}$. Hence, $W_{t}=\left(W_{1, t}, W_{2, t}\right)$ is a 2-dimensional Brownian motion on $(\Omega, \mathcal{F}, \mathbb{P})$. Note that if we define $\widehat{W}_{t} \equiv \rho d W_{1, t}+\sqrt{1-\rho^{2}} d W_{2, t}$ then $W_{1, t}$ is correlated with $\widehat{W}_{t}$ and their correlation coefficient is $\rho \in(-1,1)$, i.e., $\left\langle d W_{1, t}, d \widehat{W}_{t}\right\rangle=\rho d t$. Let $\left(\mathcal{F}_{t}\right)_{t \geq 0}$ be the $\mathbb{P}$-augmentation of the natural filtration generated by $W_{t}=\left(W_{1, t}, W_{2, t}\right)$.

Now let us denote by

$$
\begin{aligned}
& \pi_{0, t} \text {-proportion of wealth invested in the riskless asset, } \\
& \pi_{1, t} \text {-proportion of wealth invested in the risky asset, } \\
& \pi_{2, t} \text {-proportion of wealth invested in inflation-linked index bond, } \\
& c_{t}^{N} \text {-nominal consumption rate, } \\
& y_{t}^{N} \text {-nominal income rate. }
\end{aligned}
$$

Denote by $X_{t}^{N}$ the nominal value of the wealth. Then we have

$$
d X_{t}^{N}=\pi_{0, t} X_{t}^{N} \frac{d B_{t}}{B_{t}}+\pi_{1, t} X_{t}^{N} \frac{d S_{t}}{S_{t}}+\pi_{2, t} X_{t}^{N} \frac{d I_{t}}{I_{t}}-c_{t}^{N} d t+y^{N} d t
$$


The inflation-adjusted real wealth level $X_{t}$ is given by $X_{t}=X_{t}^{N} / P_{t}$. We assume that the nominal income rate $y_{t}^{N}$ is proportional to the price level $P_{t}$, i.e.,

$$
y_{t}^{N}=y P_{t}
$$

for some constant $y>0$. If we apply Itô's product rule to $X_{t}^{N} / P_{t}$, we obtain the inflationadjusted real wealth process as in the following lemma.

\section{Lemma 1}

$$
\begin{aligned}
d X_{t}= & \left(r X_{t}-c_{t}+y\right) d t+\pi_{0, t} X_{t}\left\{\left(R-r-\mu_{P}+\sigma_{P}^{2}\right) d t-\rho \sigma_{P} d W_{1, t}-\sqrt{1-\rho^{2}} \sigma_{P} d W_{2, t}\right\} \\
& +\pi_{1, t} X_{t}\left\{\left(\mu_{S}-r-\mu_{P}+\sigma_{P}^{2}-\rho \sigma_{P} \sigma_{S}\right) d t\right. \\
& \left.+\left(-\rho \sigma_{P}+\sigma_{S}\right) d W_{1, t}-\sqrt{1-\rho^{2}} \sigma_{P} d W_{2, t}\right\}
\end{aligned}
$$

where $c_{t} \equiv c_{t}^{N} / P_{t}$ is the inflation-adjusted real consumption rate, and $y$ is the inflationadjusted real income rate.

Proof

$$
d\left(\frac{1}{P_{t}}\right)=-\frac{1}{P_{t}}\left\{\left(\mu_{P}-\sigma_{P}^{2}\right) d t+\rho \sigma_{P} d W_{1, t}+\sqrt{1-\rho^{2}} \sigma_{P} d W_{2, t}\right\} .
$$

We apply Itô's product rule to get

$$
\begin{aligned}
d\left(X_{t}^{N} / P_{t}\right)= & P_{t} d X_{t}^{N}+X_{t}^{N} d P_{t}+d X_{t}^{N} d\left(\frac{1}{P_{t}}\right) \\
= & {\left[-\left(\mu_{P}-\sigma_{P}^{2}\right) X_{t}-c_{t}+y+R \pi_{0, t} X_{t}+\left(\mu_{S}-\rho \sigma_{P} \sigma_{S}\right) \pi_{1, t} X_{t}\right.} \\
& \left.+\left(r+\mu_{P}-\sigma_{P}^{2}\right) \pi_{2, t} X_{t}\right] d t \\
& +\left(-\rho \sigma_{P} X_{t}+\sigma_{S} \pi_{1, t} X_{t}+\rho \sigma_{P} \pi_{2, t} X_{t}\right) d W_{1, t} \\
& +\sqrt{1-\rho^{2}} \sigma_{P}\left(-X_{t}+\pi_{2, t} X_{t}\right) d W_{2, t} .
\end{aligned}
$$

Rearranging (2.2) with $\pi_{0, t}+\pi_{1, t}+\pi_{2, t}=1$ yields the inflation-adjusted real wealth process (2.1).

Let us define

$$
\theta_{1} \equiv \frac{\mu_{S}-R-\rho \sigma_{P} \sigma_{S}}{\sigma_{S}}, \quad \theta_{2} \equiv \frac{r-R+\mu_{P}-\sigma_{P}^{2}}{\sigma_{P} \sqrt{1-\rho^{2}}}-\frac{\rho}{\sqrt{1-\rho^{2}}} \theta_{1}
$$

an exponential martingale

$$
\xi_{t} \equiv e^{-\frac{1}{2}\left(\theta_{1}^{2}+\theta_{2}^{2}\right) t-\theta_{1} d W_{1, t}-\theta_{2} d W_{2, t}},
$$

and the pricing kernel

$$
H_{t} \equiv e^{-r t} \xi_{t}
$$


Then by the Girsanov's theorem, for any fixed $T$, there exists an equivalent martingale probability measure $\widetilde{\mathbb{P}}$ defined by $\widetilde{\mathbb{P}}(A)=\mathbb{E}\left[\xi_{T} \mathbf{1}_{A}\right], A \in \mathcal{F}_{T}$, and under the new probability measure $\widetilde{\mathbb{P}}$,

$$
\widetilde{W}_{1, t} \equiv \theta_{1} t+W_{1, t}, \quad \widetilde{W}_{2, t} \equiv \theta_{2} t+W_{2, t},
$$

are independent standard Brownian motions. Applying Itô's lemma to the product of $e^{-r t}$ and $X_{t}$ yields

$$
\begin{aligned}
d\left(e^{-r t} X_{t}\right)= & e^{-r t}\left(-c_{t}+y\right) d t+e^{-r t}\left(\pi_{0, t} X_{t}\right)\left\{-\rho \sigma_{P} d \widetilde{W}_{1, t}-\sqrt{1-\rho^{2}} \sigma_{P} d \widetilde{W}_{2, t}\right\} \\
& +e^{-r t}\left(\pi_{1, t} X_{t}\right)\left\{\left(-\rho \sigma_{P}+\sigma_{S}\right) d \widetilde{W}_{1, t}-\sqrt{1-\rho^{2}} \sigma_{P} d \widetilde{W}_{2, t}\right\} .
\end{aligned}
$$

Integrating (2.3) from 0 to $T>0$ we obtain

$$
\begin{aligned}
e^{-r T} X_{t}+\int_{0}^{T} e^{-r t}\left(c_{t}-y\right) d t= & x+\int_{0}^{T} e^{-r t} X_{t}\left\{-\rho \sigma_{P} \pi_{0, t}+\left(-\rho \sigma_{P}+\sigma_{S}\right) \pi_{1, t}\right\} d \widetilde{W}_{1, t} \\
& -\int_{0}^{T} e^{-r t} X_{t} \sqrt{1-\rho^{2}}\left(\pi_{0, t}+\pi_{1, t}\right) d \widetilde{W}_{2, t} .
\end{aligned}
$$

If $X_{t}, t>0$, is lower bounded, the right-hand side of (2.4) is a lower-bounded local martingale under $\widetilde{\mathbb{P}}$, hence a supermartingale under $\widetilde{\mathbb{P}}$. We then obtain

$$
\widetilde{\mathbb{E}}\left[e^{-r T} X_{T}+\int_{0}^{T} e^{-r T}\left(c_{t}-y\right) d t\right] \leq x,
$$

and if we take the limit $T \rightarrow \infty$ and recover the physical measure by applying Bayes' rule, the static budget constraint given in (2.5) is transformed into the following inequality:

$$
\mathbb{E}\left[\int_{0}^{\infty} H_{t} c_{t} d t\right] \leq x+\mathbb{E}\left[\int_{0}^{\infty} H_{t} y d t\right]
$$

\section{Optimization problem}

We consider the agent's expected utility maximization problem where the utility function is quadratic as follows:

$$
u(c)=c-Q c^{2}, \quad Q>0 .
$$

Notice that the quadratic utility function has its global maximum at $\bar{c} \equiv 1 / 2 Q$ which is called a bliss point. Obviously, the utility function is symmetric with respect to that point, which means the utility is increasing for $c<\bar{c}$ and decreasing for $c>\bar{c}$ and the values of the utility at $\hat{c}=c<\bar{c}$ and $1 / Q-\hat{c}$ are equivalent. Moreover, the constant $Q$ reflects the agent's risk aversion. In particular, a larger (or smaller) $Q$ implies a smaller (or larger) marginal utility if the consumption rate is smaller than the bliss point. In the mean-variance analysis, $Q$ can be regraded as a risk aversion parameter measured by the variance of wealth.

Definition 1 We call $\boldsymbol{c} \equiv\left(c_{t}\right)_{t \geq 0}$ and $\pi \equiv\left(\pi_{0, t}, \pi_{1, t}, \pi_{2, t}\right)_{t \geq 0}$ the consumption and portfolio process, respectively. We call $(\boldsymbol{c}, \boldsymbol{\pi})$ an admissible policy at $x$ if 
(a) $X_{t}$ evolves according to (2.1), $X_{0}=x$, and $X_{t}>-\frac{y}{r}, t \geq 0$,

(b) $\boldsymbol{c}$ is a measurable, adapted nonnegative process, and $\int_{0}^{t} c_{s} d s<\infty$, for all $t \geq 0$ a.s.,

(c) $\pi$ is a measurable, adapted process and $\int_{0}^{t}\left(\pi_{0, s}^{2}+\pi_{1, s}^{2}\right) d s<\infty$ for all $t \geq 0$ a.s.

Denote by $\mathcal{A}(x)$ the set of all admissible policies at $x$. Then our optimization problem is stated as follows.

Problem 1 The agent wants to maximize the expected utility by optimally choosing the consumption and portfolio processes. In other words,

$$
V(x) \equiv \max _{(\boldsymbol{c}, \pi) \in \mathcal{A}(x)} J_{(\boldsymbol{c}, \pi)}(x)
$$

where

$$
J_{(\mathbf{c}, \pi)}(x) \equiv \mathbb{E}\left[\int_{0}^{\infty} e^{-\beta t} u\left(c_{t}\right) d t\right]
$$

subject to the budget constraint (2.6); $\beta(>0)$ is the time preference of the agent.

Let us define a convex dual function $\widetilde{u}(\lambda)$ as follows:

$$
\widetilde{u}(\lambda) \equiv \max _{c>0}[u(c)-\lambda c]=\max _{c>0}\left[(1-\lambda) c-Q c^{2}\right]=\frac{(1-\lambda)^{2}}{4 Q} \mathbf{1}_{\{0<\lambda \leq 1\}} .
$$

Then the first order condition implies that the maximizer $c$ of (3.1) is given by

$$
c=\frac{1-\lambda}{2 Q} \mathbf{1}_{\{0<\lambda \leq 1\}} .
$$

Thus, for any $(\boldsymbol{c}, \boldsymbol{\pi})$ and $\lambda>0$, we have

$$
\begin{aligned}
J_{(c, \pi)}(x) & \leq \mathbb{E}\left[\int_{0}^{\infty} e^{-\beta t}\left\{\widetilde{u}\left(\lambda_{t}\right)+c_{t} \lambda_{t}\right\} d t\right] \\
& =\mathbb{E}\left[\int_{0}^{\infty} e^{-\beta t} \widetilde{u}\left(\lambda_{t}\right) d t\right]+\lambda \mathbb{E}\left[\int_{0}^{\infty} H_{t} c_{t} d t\right] \\
& \leq \mathbb{E}\left[\int_{0}^{\infty} e^{-\beta t}\left\{\widetilde{u}\left(\lambda_{t}\right)+y \lambda_{t}\right\} d t\right]+\lambda x \\
& =\mathbb{E}\left[\int_{0}^{\infty} e^{-\beta t}\left\{\frac{\left(1-\lambda_{t}\right)^{2}}{4 Q} \mathbf{1}_{\left\{0<\lambda_{t} \leq 1\right\}}+y \lambda_{t}\right\} d t\right]+\lambda x,
\end{aligned}
$$

where $\lambda_{t}=\lambda e^{\beta t} H_{t}$. Note that $\lambda_{t}$ has its dynamics as

$$
\frac{d \lambda_{t}}{\lambda_{t}}=(\beta-r) d t-\theta_{1} d W_{1, t}-\theta_{2} d W_{2, t} .
$$

If we define the dual value function $\widetilde{J}$ as

$$
\widetilde{J}(\lambda) \equiv \mathbb{E}\left[\int_{0}^{\infty} e^{-\beta t}\left\{\frac{\left(1-\lambda_{t}\right)^{2}}{4 Q} \mathbf{1}_{\left\{0<\lambda_{t} \leq 1\right\}}+y \lambda_{t}\right\} d t\right]
$$


then by Feynman-Kac's formula, $\widetilde{J}(\lambda)$ solves the following ordinary differential equation (ODE):

$$
\begin{cases}\frac{1}{2}\left(\theta_{1}^{2}+\theta_{2}^{2}\right) \lambda^{2} \widetilde{J}^{\prime \prime}(\lambda)+(\beta-r) \lambda \widetilde{J}^{\prime}(\lambda)-\tilde{\beta J}(\lambda)+\frac{(1-\lambda)^{2}}{4 Q}+y \lambda=0, & 0<\lambda \leq 1, \\ \frac{1}{2}\left(\theta_{1}^{2}+\theta_{2}^{2}\right) \lambda^{2} \widetilde{J}^{\prime \prime}(\lambda)+(\beta-r) \lambda \widetilde{J}^{\prime}(\lambda)-\beta \widetilde{J}(\lambda)+y \lambda=0, & \lambda>1 .\end{cases}
$$

Let us denote by $n_{+}$and $n_{-}$, respectively, the positive and negative roots of the equation

$$
\frac{1}{2}\left(\theta_{1}^{2}+\theta_{2}^{2}\right) n^{2}+\left\{\beta-r-\frac{1}{2}\left(\theta_{1}^{2}+\theta_{2}^{2}\right)\right\} n-\beta=0 .
$$

Then by the growth condition, we can obtain the general closed-form solution to the ODE (3.5). The next proposition summarizes the result.

Proposition 1 The dual value function is given by

$$
\widetilde{J}(\lambda)= \begin{cases}D_{1} \lambda^{n_{+}}-\frac{1}{4 Q\left(\theta_{1}^{2}+\theta_{2}^{2}+\beta-2 r\right)} \lambda^{2}+\left(\frac{y}{r}-\frac{1}{2 Q r}\right) \lambda+\frac{1}{4 Q \beta}, & 0<\lambda \leq 1, \\ D_{2} \lambda^{n_{-}}+\frac{y}{r} \lambda, & \lambda>1,\end{cases}
$$

where $D_{1}$ and $D_{2}$ are given by

$$
D_{1}=\frac{\frac{2-n_{-}}{4 \mathrm{Q}\left(\theta_{1}^{2}+\theta_{2}^{2}+\beta-2 r\right)}+\frac{1-n_{-}}{2 Q r}+\frac{n_{-}}{4 Q \beta}}{n_{+}-n_{-}}, \quad D_{2}=D_{1}-\frac{1}{4 Q\left(\theta_{1}^{2}+\theta_{2}^{2}+\beta-2 r\right)}-\frac{1}{2 Q r}+\frac{1}{4 Q \beta} .
$$

We assume that the following inequality holds.

\section{Assumption 1}

$$
\beta-2 r+\theta_{1}^{2}+\theta_{2}^{2}>0
$$

Under this assumption, we can show that the constants $D_{1}$ and $D_{2}$ are positive.

\section{Lemma 2 The following inequalities hold:}

$$
D_{1}>0, \quad D_{2}>0 .
$$

Proof We borrow the idea from Koo et al. [5]. Note that the constant $D_{1}$ can be rewritten as

$$
D_{1}=\frac{1}{\left(n_{+}-n_{-}\right) 2 R}\left(\frac{2-n_{-}}{2\left(\beta-2 r+\theta_{1}^{2}+\theta_{2}^{2}\right)}+\frac{1-n_{-}}{r}+\frac{n_{-}}{2 \beta}\right),
$$

so it is enough to show that the value in the parenthesis is positive. Let us define functions $f(n)$ and $g(x)$ by

$$
f(n) \equiv \frac{1}{2}\left(\theta_{1}^{2}+\theta_{2}^{2}\right)\left(n-n_{+}\right)\left(n-n_{-}\right)
$$


and

$$
g(x) \equiv-\frac{f(x)}{x-n_{-}}=-\frac{1}{2}\left(\theta_{1}^{2}+\theta_{2}^{2}\right)\left(x-n_{+}\right),
$$

respectively. Then, for $x \in\left(n_{-}, n_{+}\right), g(x)$ is positive and strictly decreasing. Thus, we have $g(0)>g(1)>0$, which implies

$$
-\frac{-\beta}{-n_{-}}>\frac{r}{1-n_{-}}>0
$$

or equivalently,

$$
\frac{1-n_{-}}{r}+\frac{n_{-}}{\beta}>0
$$

By Assumption 1, we also have

$$
\frac{2-n_{-}}{\beta-2 r+\theta_{1}^{2}+\theta_{2}^{2}}+\frac{1-n_{-}}{r}>0 .
$$

Therefore, by combining these two inequalities, we obtain

$$
\frac{2-n_{-}}{2\left(\beta-2 r+\theta_{1}^{2}+\theta_{2}^{2}\right)}+\frac{1-n_{-}}{r}+\frac{n_{-}}{2 \beta}>0,
$$

from which we have $D_{1}>0$.

To show the positivity of $D_{2}$, first note that $D_{2}$ can be rewritten as

$$
D_{2}=\frac{1}{\left(n_{+}-n_{-}\right) 2 R}\left(\frac{2-n_{+}}{2\left(\beta-2 r+\theta_{1}^{2}+\theta_{2}^{2}\right)}+\frac{1-n_{+}}{r}+\frac{n_{+}}{2 \beta}\right) .
$$

Similarly to the proof of the positivity of $D_{1}$, let us define function $k(x)$ by

$$
k(x) \equiv \frac{x-n_{-}}{f(x)}=\frac{2}{\left(\theta_{1}^{2}+\theta_{2}^{2}\right)} \frac{1}{x-n_{-}} .
$$

Then for $x>n_{-}, k(x)$ is positive, strictly decreasing and convex. Further, convexity implies

$$
k(1)=k\left(0 \cdot \frac{1}{2}+2 \cdot \frac{1}{2}\right)<\frac{1}{2}(k(0)+k(2)),
$$

or equivalently,

$$
\frac{1-n_{+}}{f(1)} \leq \frac{-n_{+}}{2 f(0)}+\frac{2-n_{-}}{2 f(2)} .
$$

Therefore, we can show that

$$
-\frac{1-n_{+}}{r} \leq \frac{n_{+}}{2 \beta}+\frac{2-n_{+}}{2\left(\beta-2 r+\theta_{1}^{2}+\theta_{2}^{2}\right)},
$$

and consequently $D_{2}>0$. 


\section{Solution}

In this section we provide the closed-form solution to Problem 1 . From the duality relation, we can recover the primal value function $V(x)$ through

$$
V(x)=\inf _{\lambda>0}[\widetilde{J}(\lambda)+\lambda x]
$$

where $\widetilde{J}(\lambda)$ is given in Proposition 1. Notice that from the optimal consumption rate in (3.2), $\lambda=0$ corresponds to the bliss level of consumption $\bar{c}$. Let us denote by $\bar{x}$ the wealth level at $\lambda=0$, which is then defined by

$$
\bar{x} \equiv-\frac{y}{r}+\frac{1}{2 Q r} .
$$

Moreover, if we denote the wealth level corresponding to $\lambda=1$ by $\tilde{x}$, it is also given by

$$
\tilde{x}=-n_{-} D_{2}-\frac{y}{r}=-n_{+} D_{1}+\frac{1}{2 Q\left(\theta_{1}^{2}+\theta_{2}^{2}+\beta-2 r\right)}-\frac{y}{r}+\frac{1}{2 Q r} .
$$

Thus, we have to separate the wealth level into three regions, which are $(-y / r, \tilde{x}),[\tilde{x}, \bar{x})$, and $[\bar{x}, \infty)$. The next theorem provides the value function on each region. Before we proceed, let us define

$$
h_{1}(z) \equiv-n_{+} D_{1} z^{n_{+}}+\frac{1}{2 Q\left(\theta_{1}^{2}+\theta_{2}^{2}+\beta-2 r\right)} z-\frac{y}{r}+\frac{1}{2 Q r},
$$

and

$$
h_{2}(z) \equiv-n_{-} D_{2} z^{n_{-}-1}-\frac{y}{r} .
$$

Theorem 1 The value function of Problem 1 is given by

$$
V(x)= \begin{cases}D_{2}\left(\lambda^{* *}\right)^{n_{-}}+\frac{y}{r} \lambda^{* *}+x \lambda^{* *}, & -\frac{y}{r}<x<\tilde{x}, \\ D_{1}\left(\lambda^{*}\right)^{n_{+}}-\frac{1}{4 Q\left(\theta_{1}^{2}+\theta_{2}^{2}+\beta-2 r\right)}\left(\lambda^{*}\right)^{2}+\left(\frac{y}{r}-\frac{1}{2 Q r}\right) \lambda^{*}+\frac{1}{4 Q \beta}+x \lambda^{*}, & \tilde{x} \leq x<\bar{x}, \\ \frac{1}{4 Q \beta}, & x \geq \bar{x},\end{cases}
$$

where $\lambda^{* *}$ and $\lambda^{*}$ solve $x=h_{2}\left(\lambda^{* *}\right)$ for $\frac{y}{r}<x<\tilde{x}$ and $x=h_{1}\left(\lambda^{*}\right)$ for $\tilde{x} \leq x<\bar{x}$.

Proof If we substitute the dual value function $\widetilde{J}(\lambda)$ given in Proposition 1 into the duality relation in (4.1), the relations between the inflation-adjusted real wealth level $x$ and its dual variables $\lambda^{*}$ and $\lambda^{* *}$ can be obtained from the first order conditions. It is obvious that with the inflation-adjusted real wealth level $x$ above $\bar{x}$, which corresponds to the bliss level of consumption $\bar{c}$, it is optimal to consume at a rate $\bar{c}$, and consequently the value function constantly equals to

$$
V(x)=\int_{0}^{\infty} e^{-\beta t}\left(\bar{c}-Q \bar{c}^{2}\right) d t=\frac{1}{4 Q \beta} .
$$

It is worth to note that the first region, $-y / r<x<\tilde{x}$, corresponds to $\lambda>1$ and in that region the optimal consumption rate is zero. Thus, the agent endures zero consumption 
until when the wealth level is greater than $\tilde{x}$. The optimal consumption rate is asymmetric with respect to the bliss point even though the utility function is symmetric. We summarize the optimal consumption and portfolios in the next theorem.

Theorem 2 The optimal wealth, consumption, and portfolios are given as follows:

(1) For $-\frac{y}{r}<X_{t}<\tilde{x}$,

$$
\left\{\begin{array}{l}
X_{t}=-n_{-} D_{2}\left(\lambda_{t}^{* *}\right)^{n_{-}-1}-\frac{y}{r}, \\
c_{t}^{*}=0, \\
\pi_{0, t}^{*} X_{t}=\left\{-\frac{\theta_{1}}{\sigma_{S}}+\left(\frac{\rho}{\sigma_{S}}-\frac{1}{\sigma_{P}}\right) \frac{\theta_{2}}{\sqrt{1-\rho^{2}}}\right\} n_{-}\left(n_{-}-1\right) D_{2}\left(\lambda_{t}^{* *}\right)^{n_{-}-1}, \\
\pi_{1, t}^{*} X_{t}=\left(\frac{\theta_{1}}{\sigma_{S}}-\frac{\rho \theta_{2}}{\sigma_{S} \sqrt{1-\rho^{2}}}\right) n_{-}\left(n_{-}-1\right) D_{2}\left(\lambda_{t}^{* *}\right)^{n_{-}-1}, \\
\pi_{2, t}^{*} X_{t}=X_{t}-\pi_{0, t}^{*} X_{t}-\pi_{1, t}^{*} X_{t},
\end{array}\right.
$$

where $\lambda_{t}^{* *}$ solves $X_{t}=h_{2}\left(\lambda_{t}^{* *}\right)$.

(2) For $\tilde{x} \leq X_{t}<\bar{x}$,

$$
\left\{\begin{array}{l}
X_{t}=-n_{+} D_{1}\left(\lambda_{t}^{*}\right)^{n_{+}-1}+\frac{1}{2 Q\left(\theta_{1}^{2}+\theta_{2}^{2}+\beta-2 r\right)} \lambda_{t}^{*}-\frac{y}{r}+\frac{1}{2 Q r}, \\
c_{t}^{*}=\frac{1-\lambda_{t}^{*}}{2 Q} \\
\pi_{0, t}^{*} X_{t}=\left\{-\frac{\theta_{1}}{\sigma_{S}}+\left(\frac{\rho}{\sigma_{S}}-\frac{1}{\sigma_{P}}\right) \frac{\theta_{2}}{\sqrt{1-\rho^{2}}}\right\}\left\{n_{+}\left(n_{+}-1\right) D_{1}\left(\lambda_{t}^{*}\right)^{n_{+}-1}-\frac{1}{2 Q\left(\theta_{1}^{2}+\theta_{2}^{2}+\beta-2 r\right)} \lambda_{t}^{*}\right\} \\
\pi_{1, t}^{*} X_{t}=\left(\frac{\theta_{1}}{\sigma_{S}}-\frac{\rho \theta_{2}}{\sigma_{S} \sqrt{1-\rho^{2}}}\right)\left\{n_{+}\left(n_{+}-1\right) D_{1}\left(\lambda_{t}^{*}\right)^{n_{+}-1}-\frac{1}{2 Q\left(\theta_{1}^{2}+\theta_{2}^{2}+\beta-2 r\right)} \lambda_{t}^{*}\right\}, \\
\pi_{2, t}^{*} X_{t}=X_{t}-\pi_{0, t}^{*} X_{t}-\pi_{1, t}^{*} X_{t},
\end{array}\right.
$$

where $\lambda_{t}^{*}$ solves $X_{t}=h_{1}\left(\lambda_{t}^{*}\right)$.

(3) For $X_{t} \geq \bar{x}$,

$$
\left\{\begin{array}{l}
c_{t}^{*}=\frac{1}{2 Q}, \\
\pi_{0, t}^{*} X_{t}=0, \\
\pi_{1, t}^{*} X_{t}=0, \\
\pi_{2, t}^{*} X_{t}=X_{t} .
\end{array}\right.
$$

Proof From Theorem 1, $X_{t}=h_{2}\left(\lambda_{t}^{* *}\right)=-n_{-} D_{2}\left(\lambda_{t}^{* *}\right)^{n_{-}-1}-\frac{y}{r}$ for $-\frac{y}{r}<X_{t}<\tilde{x}$. We use Itô's lemma for $X_{t}$ along with $d \lambda_{t}^{* *} / \lambda_{t}^{* *}=(\beta-r) d t-\theta_{1} d W_{1, t}-\theta_{2} d W_{2, t}$ to obtain

$$
\begin{aligned}
d X_{t}= & -n_{-} D_{2}\left[\left(n_{-}-1\right)\left(\lambda_{t}^{* *}\right)^{n_{-}-1}\left\{(\beta-r) d t-\theta_{1} d W_{1, t}-\theta_{2} d W_{2, t}\right\}\right. \\
& \left.+\frac{1}{2}\left(n_{-}-1\right)\left(n_{-}-2\right)\left(\theta_{1}^{2}+\theta_{2}^{2}\right)\left(\lambda_{t}^{* *}\right)^{n_{-}-1} d t\right] \\
= & -n_{-}\left(n_{-}-1\right) D_{2}\left\{(\beta-r)+\frac{1}{2}\left(n_{-}-2\right)\left(\theta_{1}^{2}+\theta_{2}^{2}\right)\right\}\left(\lambda_{t}^{* *}\right)^{n_{-}-1} d t \\
& +n_{-}\left(n_{-}-1\right) D_{2} \theta_{1}\left(\lambda_{t}^{* *}\right)^{n_{-}-1} d W_{1, t}+n_{-}\left(n_{-}-1\right) D_{2} \theta_{2}\left(\lambda_{t}^{* *}\right)^{n_{-}-1} d W_{2, t} \\
= & (\cdots)+\left\{-\rho \sigma_{P} \pi_{0, t} X_{t}+\left(-\rho \sigma_{P}+\sigma_{S}\right) \pi_{1, t} X_{t}\right\} d W_{1, t} \\
& -\sqrt{1-\rho^{2}} \sigma_{P}\left\{\pi_{0, t} X_{t}+\pi_{1, t} X_{t}\right\} d W_{2, t},
\end{aligned}
$$


where the last equality comes from rearranging (2.1). Comparing diffusion terms in (4.3) and (4.4), we obtain the optimal portfolios $\pi_{0, t}^{*} X_{t}$ and $\pi_{1, t}^{*} X_{t}$. Moreover, $\pi_{2, t}^{*} X_{t}$ can be found from the relation $\pi_{0, t}^{*}+\pi_{1, t}^{*}+\pi_{2, t}^{*}=1$. The optimal inflation-adjusted real consumption rate $c^{*}$ is such that makes the first equality in (3.3) hold as an equality, i.e., (3.2) is satisfied. For $-\frac{y}{r}<X_{t} \leq \tilde{x}, \lambda_{t}^{* *} \geq 1$, hence $c^{*}=0$.

Similarly, for $\tilde{x} \leq X_{t}<\bar{x}, X_{t}=h_{1}\left(\lambda_{t}^{*}\right)=-n_{+} D_{1}\left(\lambda_{t}^{*}\right)^{n_{+}-1}+\frac{1}{2 Q\left(\theta_{1}^{2}+\theta_{2}^{2}+\beta-2 r\right)} \lambda_{t}^{*}-\frac{y}{r}+\frac{1}{2 Q r}$, and we obtain

$$
\begin{aligned}
d X_{t}= & -n_{+} D_{1}\left[\left(n_{+}-1\right)\left(\lambda_{t}^{*}\right)^{n_{+}-1}\left\{(\beta-r) d t-\theta_{1} d W_{1, t}-\theta_{2} d W_{2, t}\right\}\right. \\
& \left.+\frac{1}{2}\left(n_{+}-1\right)\left(n_{+}-2\right)\left(\theta_{1}^{2}+\theta_{2}^{2}\right)\left(\lambda_{t}^{*}\right)^{n_{+}-1} d t\right] \\
& +\frac{\lambda_{t}^{*}}{2 Q\left(\theta_{1}^{2}+\theta_{2}^{2}+\beta-2 r\right)}\left\{(\beta-r) d t-\theta_{1} d W_{1, t}-\theta_{2} d W_{2, t}\right\} \\
= & (\cdots) d t+\left\{n_{+}\left(n_{+}-1\right) \theta_{1} D_{1}\left(\lambda_{t}^{*}\right)^{n_{+}-1}-\frac{\theta_{1}}{2 Q\left(\theta_{1}^{2}+\theta_{2}^{2}+\beta-2 r\right)} \lambda_{t}^{*}\right\} d W_{1, t} \\
& +\left\{n_{+}\left(n_{+}-1\right) \theta_{2} D_{1}\left(\lambda_{t}^{*}\right)^{n_{+}-1}-\frac{\theta_{2}}{2 Q\left(\theta_{1}^{2}+\theta_{2}^{2}+\beta-2 r\right)} \lambda_{t}^{*}\right\} d W_{2, t} \\
= & (\cdots)+\left\{-\rho \sigma_{P} \pi_{0, t} X_{t}+\left(-\rho \sigma_{P}+\sigma_{S}\right) \pi_{1, t} X_{t}\right\} d W_{1, t} \\
& -\sqrt{1-\rho^{2}} \sigma_{P}\left\{\pi_{0, t} X_{t}+\pi_{1, t} X_{t}\right\} d W_{2, t},
\end{aligned}
$$

from which we obtain optimal portfolios, and $c_{t}^{*}$ can be obtained from (3.2).

Lastly, the case for $X_{t} \geq \bar{x}$ can be derived by letting $\lambda_{t}^{*} \rightarrow 0$ in the case where $\widetilde{x} \leq$ $X_{t}<\bar{x}$.

For $-y / r<X_{t}<\tilde{x}$, the optimal investments in the risky asset and the inflation-linked index bond is monotone in wealth but, for $\tilde{x} \leq X_{t}<\bar{x}$, the optimal investments in the risky asset and the inflation-linked index bond have their extreme values at $\hat{\lambda}$, which is given by

$$
\hat{\lambda}=\left(\frac{1}{2 Q n_{+}\left(n_{+}-1\right)^{2}\left(\beta-2 r+\theta_{1}^{2}+\theta_{2}^{2}\right) D_{1}}\right)^{\frac{1}{n_{+}-2}} .
$$

If $\frac{1}{\sigma_{s}}\left(\theta_{1}-\frac{\rho \theta_{2}}{\sqrt{1-\rho^{2}}}\right)>0$, we can check that $\pi_{1, t}^{*} X_{t}^{*}$ is maximized at $X_{t}=\hat{x}$, where

$$
\hat{x}=-n_{+} D_{1}(\hat{\lambda})^{n_{+}-1}+\frac{1}{2 Q\left(\beta-2 r+\theta_{1}^{2}+\theta_{2}^{2}\right)} \hat{\lambda}+\frac{1}{2 r Q}-\frac{y}{r} .
$$

Furthermore, from a similar analysis in Koo et al. [5], we see that, for $\tilde{x} \leq X_{t} \leq \hat{x}, \pi_{1, t}^{*} X_{t}$ is increasing in $X_{t}$ and, for $\hat{x}<X_{t}<\bar{x}, \pi_{1, t}^{*} X_{t}$ is decreasing in $X_{t}$ and approaches zero as $X_{t}$ gets close to $\bar{x}$. Note that we have the opposite results for $\pi_{0, t}^{*} X_{t}$. Specifically, for $\tilde{x} \leq X_{t} \leq \hat{x}$, $\pi_{0, t}^{*} X_{t}$ is decreasing in $X_{t}$ and, for $\hat{x}<X_{t} \leq \bar{x}, \pi_{0, t}^{*} X_{t}$ is increasing in $X_{t}$ and approaches zero as $X_{t}$ gets close to $\bar{x}$. 


\section{Discussion}

In this section, we discuss the quantitative results obtained from the optimization problem and exhibit implications through some numerical illustrations. Let us rewrite the optimal policies as follows.

Remark 1 The optimal consumption rate in Theorem 2 can be rewritten as

$$
c_{t}^{*}= \begin{cases}0, & -y / r<X_{t}<\tilde{x}, \\ \frac{1}{2 Q}-\left(\beta-2 r+\theta_{1}^{2}+\theta_{2}^{2}\right)\left(\bar{x}-X_{t}\right) & \\ \quad+\left(\beta-2 r+\theta_{1}^{2}+\theta_{2}^{2}\right) n_{+} D_{1}\left(\lambda_{t}^{*}\right)^{n_{+}-1}, & \tilde{x} \leq X_{t}<\bar{x}, \\ \frac{1}{2 Q}, & X_{t} \geq \bar{x} .\end{cases}
$$

Similarly, the optimal portfolios can also be rewritten as

$$
\begin{aligned}
& \pi_{0, t}^{*} X_{t}= \begin{cases}-\frac{\theta_{2}\left(1-n_{-}\right)}{\sigma_{p} \sqrt{1-\rho^{2}}}\left(X_{t}+\frac{y}{r}\right)-\pi_{1, t}^{*} X_{t}, & -y / r<x_{t}<\tilde{x}, \\
-\frac{\theta_{2}}{\sigma_{p} \sqrt{1-\rho^{2}}}\left(\bar{x}-X_{t}\right)-\pi_{1, t}^{*} X_{t}, & \tilde{x} \leq X_{t}<\bar{x}, \\
0, & X_{t} \geq \bar{x},\end{cases} \\
& \pi_{1, t}^{*} X_{t}= \begin{cases}\frac{1}{\sigma_{s}}\left(\theta_{1}-\frac{\rho \theta_{2}}{\sqrt{1-\rho^{2}}}\right)\left(1-n_{-}\right)\left(X_{t}+\frac{y}{r}\right), & -y / r<x_{t}<\tilde{x}, \\
\frac{1}{\sigma_{s}}\left(\theta_{1}-\frac{\rho \theta_{2}}{\sqrt{1-\rho^{2}}}\right)\left(\bar{x}-X_{t}\right) & X_{t} \geq \bar{x}, \\
-\frac{n_{+}\left(n_{+}-2\right)}{\sigma_{s}}\left(\theta_{1}-\frac{\rho \theta_{2}}{\sqrt{1-\rho^{2}}}\right) D_{1}\left(\lambda_{t}^{*}\right)^{n_{+}-1}, & \tilde{x} \leq X_{t}<\bar{x},\end{cases} \\
& \pi_{2, t}^{*} X_{t}= \begin{cases}X_{t}+\frac{\theta_{2}\left(1-n_{-}\right)}{\sigma_{p} \sqrt{1-\rho^{2}}}\left(X_{t}+\frac{y}{r}\right), & -y / r<x_{t}<\tilde{x}, \\
X_{t}+\frac{\theta_{2}}{\sigma_{p} \sqrt{1-\rho^{2}}}\left(\bar{x}-X_{t}\right), & \tilde{x} \leq X_{t}<\bar{x}, \\
X_{t}, & X_{t} \geq \bar{x},\end{cases}
\end{aligned}
$$

respectively, where $y_{t}^{*}$ solves $X_{t}=h_{1}\left(\lambda_{t}^{*}\right)$.

Let us call $(-y / r, \tilde{x}),[\tilde{x}, \bar{x})$, and $[\bar{x}, \infty)$ as regions 1,2 , and 3 , respectively. In region 3 , as we expected, the optimal consumption rate is $1 / 2 Q$ at which the quadratic utility function has its maximum. Interestingly, we also have a constant consumption in region 1 . The main reason why the agent consumes nothing in region 1 might be that the agent wants to get out of the lower wealth level as soon as possible. This effort could be compensated as additional consumption in region 2 .

Similar to the consumption rate, the portfolios can also be described according to the different regions. We consider for the case where $\theta_{1}-\rho \theta_{2} / \sqrt{1-\rho^{2}}>0$. Then, the investment in the risky asset, $\pi_{1, t} X_{t}$, is always non-negative, and the investment in the riskless asset (or bank account), $\pi_{0,1} X_{t}$, is always non-positive. Note that $y / r$ is the present value of the future income stream, called human capital. We also call the sum of financial wealth $X_{t}$ and human capital as the total capital, which is always non-negative.

Now we can describe the optimal portfolios in more details. In region 1, the investment in the inflation-linked index bond consists of two components, which are the demand for 
hedging inflation risk and an additional investment which is proportion to the total capital. Since $n_{-}<0$, the additional investment is positive if $\theta_{2}>0$, which implies the speculative or myopic demand due to the excess return on the inflation-linked index bond. Moreover, under the condition that $\theta_{1}-\rho \theta_{2} / \sqrt{1-\rho^{2}}>0$, the investment in risky asset also represents myopic demand which is proportion to the total capital. We can verify that the total myopic demand in the risky asset and inflation-linked index bond is borrowed from the bank account.

Similar to the portfolios in region 1, in region 2 the total wealth is invested in the inflation-linked index bond to hedge the inflation risk and there exists an additional investment in that bond. The myopic demand, however, is not proportional to the total capital anymore. We see that the second term in (5.4) represents the myopic demand and it is proportional to the difference between $\bar{x}$ and current wealth. Note that $\bar{x}$ is the wealth level at bliss point. Thus, in region $2, \bar{x}$ plays a role of target wealth and a proportion $\left(\bar{x}-X_{t}\right)$ is invested as myopic demand. We can also confirm that the optimal investment in the risky asset given in (5.3) consists of two components, and the first one, which is a myopic demand, is proportional to $\left(\bar{x}-X_{t}\right)$.

Under Assumption 1, we have $n_{+}<2$. Thus, the second term of (5.3) in region 2 is positive. Since the coefficient $D_{1}$ is determined by the boundary condition at $\tilde{x}$, we can describe the second term as the hedging demand for not falling into region 1. Again, we can verify that, except for the inflation risk hedging demand, the total investment in the risky asset and the inflation-linked index bond is borrowed from the bank account as we can see in the portfolio $\pi_{0, t} X_{t}$ in (5.2).

In region 3 , the investment in the risky asset and bank account becomes zero and all the financial wealth is invested in the inflation-linked index bond. This is a quite different result from the case with other types of utility function such as CRRA or CARA. Since the quadratic utility has a bliss point and the value function is flat for wealth levels that are larger than bliss level of wealth, there should be no speculative motive, and thus, there is no myopic demand in asset allocation. In particular, we have the myopic demand in both the risky asset and the inflation-linked index bond as explained before. They converge to zero in region 3. Moreover, the hedging demand for not falling into the region 1 , which is the last term of the portfolio (5.3) in region 2, also converges to zero in region 3 . This is because the financial wealth is large enough. Recall that in region 2 , except for the inflation risk hedging demand, all the myopic and hedging demand is borrowed from the bank account. Thus, the investment in the bank account, $\pi_{0, t} X_{t}$, should be also zero and, as a result, an inflation-risk hedging demand exists only in region 3 . To sum up, in the presence of inflation risk and the inflation-linked index bond, there exists no demand in the risky asset or in the bank account. Instead, only inflation risk hedging demand remains. Neither myopic demand nor other hedging demand is necessary.

We consider the following market parameter set to for numerical illustrations:

$$
\begin{aligned}
& \mu_{S}=0.08, \quad \sigma_{S}=0.2, \quad \mu_{P}=0.023, \quad \sigma_{P}=0.05, \quad \rho=-0.07, \\
& r=0.02, \quad R=0.04, \quad y=0.5, \quad \beta=0.07, \quad Q=0.3 .
\end{aligned}
$$

This parameter set is consistent with Brennan and Xia [1], Koo et al. [5], and Kwak and Lim [6], and we have $\theta_{1}-\rho \theta_{2} / \sqrt{1-\rho^{2}}=0.2052>0$. Figure 1 shows the optimal consumption and portfolios in the presence of inflation risk. For the given parameter set, $-y / r=-25$, 

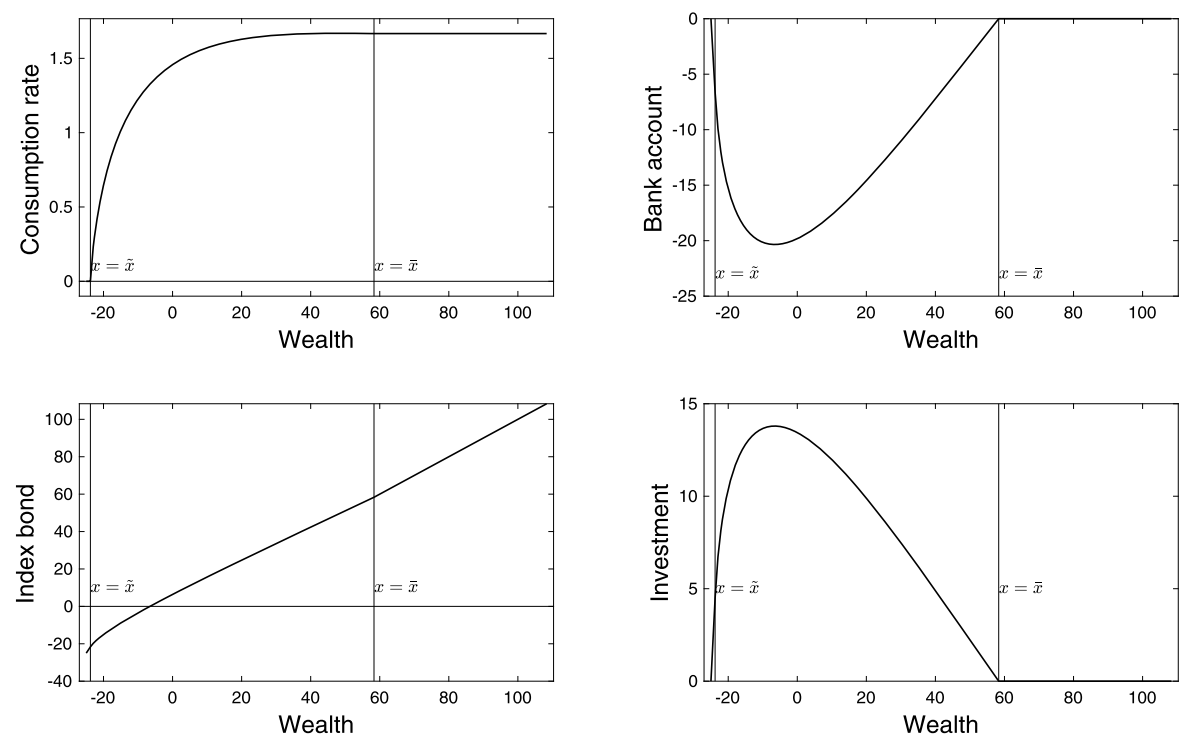

Figure 1 Optimal consumption rate and portfolios when $\theta_{1}-\rho \theta_{2} / \sqrt{1-\rho^{2}}>0$

$\tilde{x}=-23.8$ and $\bar{x}=58.3$. So regions 1,2 , and 3 are given by $(-25,-23.8),[-23.8,58.3)$, and $[58.3, \infty)$, respectively. As we can see, the consumption rate is zero in region 1 , and is strictly increasing and concave in wealth in region 2 . In region 3 , however, it stays constant. The investment in the risk asset is always non-negative and has the global maximum value at $x=\hat{x}(=-6.526)$, which is defined in (4.7). Specifically, the investment in the risky asset increases with wealth for $x \in(-y / r, \hat{x})$ and decreases with wealth for $x \in(\hat{x}, \bar{x}]$. It approaches zero as wealth gets close to $\bar{x}$. Investment in the bank account has exactly the opposite pattern compared to the investment in the risky asset. The investment in the inflation-linked index bond, however, increases with wealth in all the regions and it has the same value as the wealth when $x \geq \bar{x}$.

Now we consider another set of market parameters such that $\theta_{1}-\rho \theta_{2} / \sqrt{1-\rho^{2}}<0$. With other parameters fixed, let us consider the case of $\mu_{S}=0.5, \mu_{P}=0.035$ and $\rho=0.3$. Then we have $\theta_{1}-\rho \theta_{2} / \sqrt{1-\rho^{2}}=-0.044<0, \tilde{x}=-23.36$, and $\bar{x}=58.3$. Therefore, regions 1,2 , and 3 are given by $(-25,-23.36),[-26.36,58.3)$ and $[53.8, \infty)$, respectively. Note that $\tilde{x}$ and $\bar{x}$ have similar values as in the case with market parameters of Fig. 1. Figure 2 illustrates the optimal consumption and portfolios with these parameters. As we can see, the optimal consumption rate is similar to that of Fig. 1. The portfolios, however, have quite different results. In particular, the investment in the risky asset is not positive anymore, and it shows a similar shape to the investment in the bank account. Instead, compared to the case of Fig. 1, the investment in the inflation-linked index bond has much larger values in region 2. In fact, the negative sign of $\theta_{1}-\rho \theta_{2} / \sqrt{1-\rho^{2}}$ implies a high mean return of the inflation-linked index bond, so more speculative demand would be required. Thus, in regions 1 and 2, the agent borrows money from the bank account or takes a short position in the risky asset to finance, and all the borrowed money is invested in the inflation-linked index bond. In other words, the investment in the inflation-linked index bond consists of two different demands, which are inflation risk hedging demand and myopic demand. We observe that the myopic demand does not appear in other portfolios. Note that in region 3 , the investment in the inflation-linked index bond has the same value as the wealth level. 

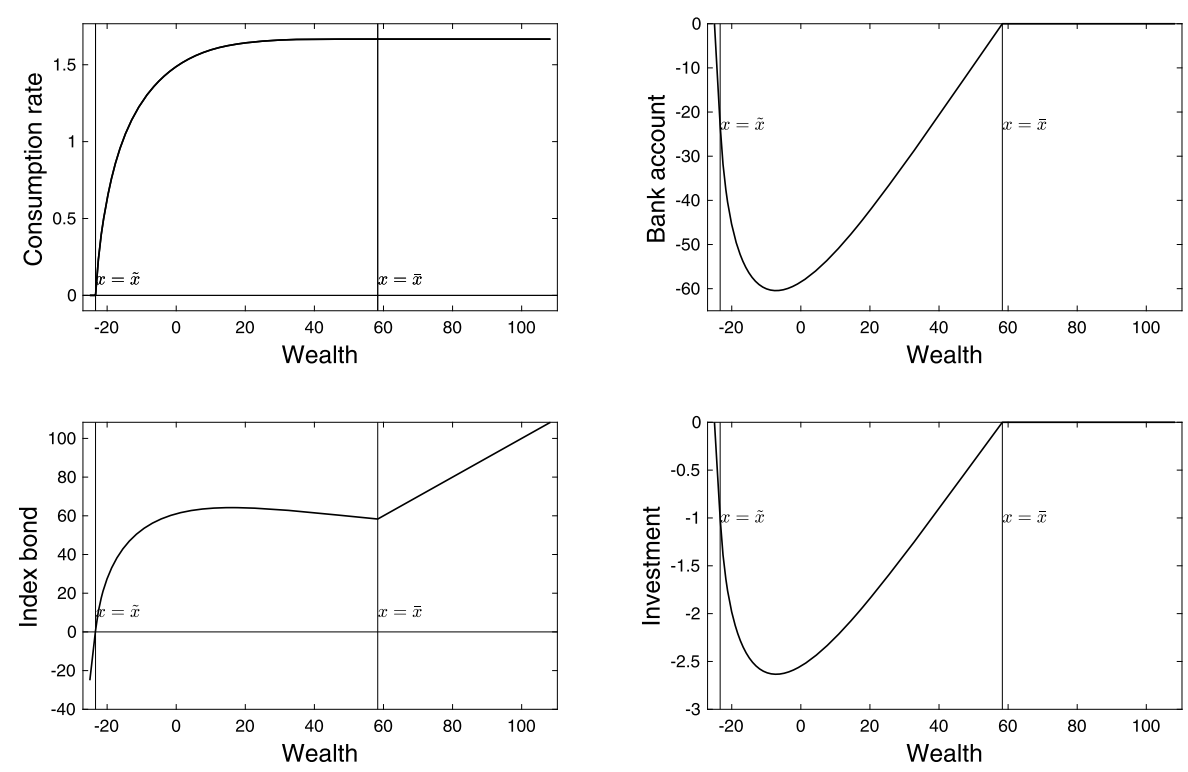

Figure 2 Optimal consumption rate and portfolios when $\theta_{1}-\rho \theta_{2} / \sqrt{1-\rho^{2}}<0$

\section{Conclusions}

In this paper, we study the optimal consumption and portfolio decision with a quadratic utility in the presence of inflation risk. For a hedging instrument for inflation risk, we introduce an inflation-linked index bond which is directly linked to the price process. Depending on market parameters, the investment in the risk asset and in the inflation-linked index bond exhibit quite different patterns. For the life-time financial planning, the inflation risk is easily ignored but it may frustrate an agent which consumes and invests optimally. Therefore, it is worthwhile to look into how an agent manages the inflation risk in the presence of an inflation-linked index bond through lifetime utility maximization with a quadratic utility function.

Acknowledgements

We are grateful to Yong Hyun Shin for helpful comments and suggestions.

\section{Funding}

Lim was supported by the National Research Foundation of Korea Grant funded by the Korean Government (NRF-2014S1A5A8018920, NRF-2017R1E1A1A03071107). Lee was supported by the National Research Foundation of Korea Grant funded by the Korean Government (NRF-2016R1D1A1B03933406) and by the Research Grant of Kwangwoon University in 2018.

Competing interests

The authors declare that they have no competing interests.

Authors' contributions

All authors contributed equally to the writing of this paper. All authors read and approved the final manuscript.

\section{Author details}

${ }^{1}$ Department of Economics and Finance, The University of Suwon, Hwaseong, Republic of Korea. ${ }^{2}$ Department of Mathematics, Kwangwoon University, Seoul, Republic of Korea.

\section{Publisher's Note}

Springer Nature remains neutral with regard to jurisdictional claims in published maps and institutional affiliations. 
References

1. Brennan, M.J., Xia, Y.: Dynamic asset allocation under inflation. J. Finance 57, 1201-1238 (2002)

2. Campbell, J.Y., Viceira, L.M.: Who should buy long-term bonds? Am. Econ. Rev. 91, 99-127 (2001). https://doi.org/10.1257/aer.91.1.99

3. Fischer, S.: The demand for index bonds. J. Polit. Econ. 83, 509-534 (1975)

4. Gong, N., Li, T.: Role of index bonds in an optimal dynamic asset allocation model with real subsistence consumption. Appl. Math. Comput. 174, 710-731 (2006)

5. Koo, J.L., Ahn, S.R., Koo, B.L., Koo, H.K., Shin, Y.H.: Optimal consumption and portfolio selection with quadratic utility and a subsistence consumption constraint. Stoch. Anal. Appl. 34, 165-177 (2016). https://doi.org/10.1080/07362994.2015.1112748

6. Kwak, M., Lim, B.H.: Optimal portfolio selection with life insurance under inflation risk. J. Bank. Finance 46, 59-71 (2014)

7. Markowitz, H.M.: Portfolio selection. J. Finance 7, 77-91 (1952). https://doi.org/10.1111/j.1540-6261.1952.tb01525.x

8. Rho, K., Kim, J.Y., Shin, Y.H.: An optimal consumption and investment problem with quadratic utility and negative wealth constraints. J. Inequal. Appl. 2017(1), Article ID 188 (2017). https://doi.org/10.1186/s13660-017-1469-X

9. Sharpe, W.F.: Expected utility asset allocation. Financ. Anal. J. 63, 18-30 (2007)

\section{Submit your manuscript to a SpringerOpen ${ }^{\circ}$ journal and benefit from:}

- Convenient online submission

Rigorous peer review

Open access: articles freely available online

High visibility within the field

- Retaining the copyright to your article

Submit your next manuscript at $\boldsymbol{\nabla}$ springeropen.com 